Pneumologe 2012 - 9:415-415

DOI 10.1007/s10405-012-0585-z

Online publiziert: 26. September 2012

(c) Springer-Verlag Berlin Heidelberg 2012

F.J.F Herth $\cdot$ M. Kreuter

Thoraxklinik, Universitätsklinikum Heidelberg

\title{
Transthorakale Sonographie
}

auch in der Pneumologie etabliert und sowohl Indikationen als auch therapeutische Möglichkeiten unter ultraschallgestützter Kontrolle nehmen einen deutlich breiteren Raum ein. Nimmt man z. B. den Pleuraerguss, so sind heutzutage die Diagnostik und die therapeutische Punktion ohne diese Art der Bildunterstützung nicht mehr denkbar.

\section{D) Die Erfahrung des Untersuchers und das kontinuierliche Lernen spielen eine große Rolle}

Trotzdem bestehen bei der Songraphie weiterhin Einschränkungen. So ist ihre Effektivität deutlich von der Erfahrung des Untersuchers abhängig. Es bedarf deshalb des kontinuierlichen Lernens in Bezug auf Technik und Darstellung der unterschiedlichen Indikationen und möglichen Komplikationen.

In dieser Ausgabe von Der Pneumologe werden die verschiedenen Methoden in 5 Übersichtsarbeiten dezidiert beschrieben. Gompelmann widmet sich in ihrem Beitrag dem Stellenwert der Technik beim Bronchialkarzinom. Insbesondere die endoluminalen Techniken werden beleuchtet. Müller stellt Indikationen, Vorgehen, aber auch Komplikationen der ultraschallgeführten Interventionen dar. Kreuter, Gasteditor dieses Heftes, widmet sich der Sonographie in der pneumologischen Notfalltherapie, Reißig der Pneumonie und den Lungengewebserkrankungen. „Last, but not least“ werden von Schuler Pleuraerguss und pleuraständige Tumoren diskutiert.
Alle Artikel sind von führenden Experten auf dem Gebiet der transthorakalen Sonographie geschrieben und die neuesten Literaturstellen werden zitiert. Allen Autoren sei an dieser Stelle gedankt für die guten Arbeiten und die Zeit, die beim Erstellen der Arbeit investiert wurde.
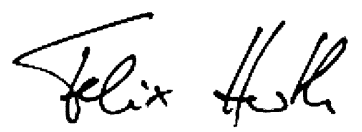

Prof. Dr. Felix J.F. Herth

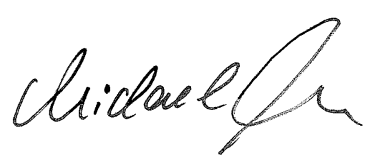

PD Dr. Michael Kreuter

\section{Korrespondenzadresse}

\section{Prof. Dr. F.J.F. Herth}

Thoraxklinik, Universitätsklinikum Heidelberg Amalienstr. 5, 69126 Heidelberg

felix.herth@thoraxklinik-heidelberg.de

Interessenkonflikt. Der korrespondierende Autor gibt für sich und seinen Koautor an, dass kein Interessenkonflikt besteht. 